Z. klin. Chem. u. klin. Biochem.

7. Jg., S. $361-364$, Juli 1969

\title{
Intoxikation bei der Blutzuckerbestimmung mit der o-Toluidin-Methode?
}

\author{
Von W.-D. Thomitzek und H. BEmm \\ Aus dem Hauptlaboratorium des Stadterankenbauses Friesenstraße (Ärztlicher Direktor: Prof. Dr. med. babil. D. Lobmann)
}

(Eingegangen am 18. März 1969)

Das für seine hohe Toxizität bekannte o-Toluidin kann aus stark saurer Lösung beim Erhitzen nicht in toxikologisch bedeutsamem Maß flüchtig werden. Ebensowenig waren Anzeichen für eine Giftwirkung vorhanden, wenn die stark essigsaure Lösung der organischen Base auf die Haut gebracht wird. Damit werden die kürzlich in der Literatur geäußerten Bedenken gegen die Verwendung der einfachen, genauen, billigen und daher weit verbreiteten o-Toluidin-Eisessig-Methode zur Blutzuckerbestimmung zerstreut.

\section{The question of toxicosis during the determination of blood sugar by the o-toluidine method}

o-Toluidine, which is known for its high toxicity, is not vaporised in toxicologically significant amounts when strong acidic solutions are heated. Similarly, there was no evidence of toxic action when a strong solution of the base in glacial acetic acid was placed on the skin. Thus recent reports in the literature regarding the danger of this simple, accurate, cheap and therefore widely used o-toluidine-acetic acid method for the determination of blood sugar, are invalid.

Die Blutzuckerbestimmung im Kliniklabor erfuhr einen entscheidenden Fortschritt, als HuLtman (1) 1959 die Bildung eines Farbkomplexes zwischen 0 -Toluidin und Aldohexosen beschrieb. Damit war die Grundlage für eine Methode geschaffen, die in zahlreich modifizierter Form $(2,3,4)$ heute aus den medizinischen Laboratorien kaum noch wegzudenken ist. Sie vereint eine hohe Genauigkeit und große Einfachheit mit einem im Vergleich zu den Enzymtests geringen Preis und ist im Blut auch von einer für praktische Belange völlig ausreichenden Spezifität. In Ausnahmefällen wird man berücksichtigen müssen, daß neben Glucose andere Aldosen wie z. B. Galaktose und Mannose reagieren können.

Es mag angesichts der vielen Vorteile und der daraus resultierenden weiten Verbreitung dieser Methode sicher vielerorts mit Aufmerksamkeit registriert worden sein, daß kürzlich BürgI und MitTELHOlZER (5) die 0 -Toluidin-Methode vor allem wegen der hohen Toxizität des 0 -Toluidin für den Routinebetrieb ablehnten. Als Prophylaxe wird das Tragen von Handschuhen zur Vermeidung des Hautkontaktes bzw. das Arbeiten in einer geschlossenen "Kapelle“ zur Verhütung der Einatmung der "giftigen Dämpfe" empfohlen, womit die Methode kaum noch diskutabel wäre.

Wir sind der Frage der Toxizität des o-Toluidins nachgegangen, da. es uns auf Grund bekannter chemischer Tatsachen sehr unwahrscheinlich erschien, daß oToluidin in der verwendeten Lösung (etwa 5-10proz. in Eisessig) von nennenswerter Toxizität sein kann. Wir haben einmal die Mengen von o-Toluidin gemessen, die bei den üblichen Versuchsbedingungen in die Luft übertreten ünd zum anderen in Tierversuchen die akute Toxizität getestet und geprüft, ob eine Hautresorption in toxikologisch bedeutsamen Ausmaß festgestellt werden kann. Als Gradmesser für die Toxizität diente der Met-Hämoglobingehalt des Blutes, da o-
Toluidin als starker Met-Hämoglobinbildner bekannt ist $(6,7)$. Zusätzlich haben wir mehrfach bei der Laborantin, die in größeren Serien täglich die Blutzuckerbestimmung nach der 0 -Toluidin-Methode durchführt, das Met-Hämoglobin gemessen.

\section{Methodik}

Bestimmung der Flüchtigkeit von 0 -Toluidin

Die Versuchsanordnung ist in Abbildung 1 dargestellt. Mit Hilfe einer Wasserstrahlpumpe wird die Luft über dem mit Toluidin gefüllten Reagenzglas abgesaugt und durch eine Vorlage von

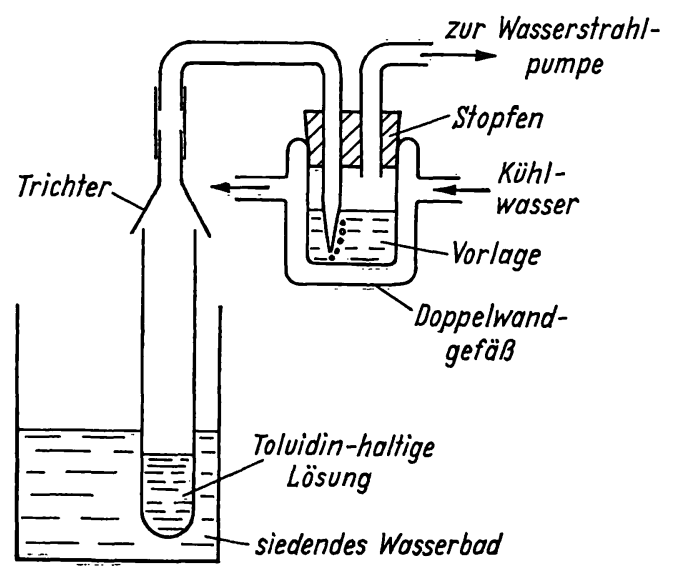

Abb. 1

Schema der Versuchanordnung zum Messen der Flüchtigkeit von o-Toluidin beim Erhitzen im siedenden Wasserbad

$0,5 \mathrm{~N}$ Essigsäure, die wassergekühlt wird, geleitet. Das von der Essigsäure gebundene Toluidin bestimmten wir quantitativ mit einer neu ausgearbeiteten Methode, die auf der bekannten Farbreaktion des $o$-Toluidin mit Natriumhypochlorit und Ammoniak beruht (vgl. (8)). Die Empfindlichkeit beträgt etwa $2 \mu \mathrm{g} / \mathrm{m} /$ Probe.

\section{Meßansatz}

$2,5 \mathrm{ml}$ Probe (5-100 $\mu \mathrm{g}$ Toluidin enthaltend) werden mit $1 \mathrm{~N}$ $\mathrm{NaOH}$ neutralisiert und auf $4 \mathrm{~m} / \mathrm{mit} \mathrm{H}_{2} \mathrm{O}$ aufgefüllt. Dann werden $0,25 \mathrm{~m} / \mathrm{NaOCl}$-Lösung $(0,14 \mathrm{proz}$. an aktivem Chlor, bestimmt nach (9)) und nach $5 \mathrm{Min} .0,25 \mathrm{~m} / 1 \mathrm{~N} \mathrm{NH}_{3}$ hinzugefügt. Die sich 


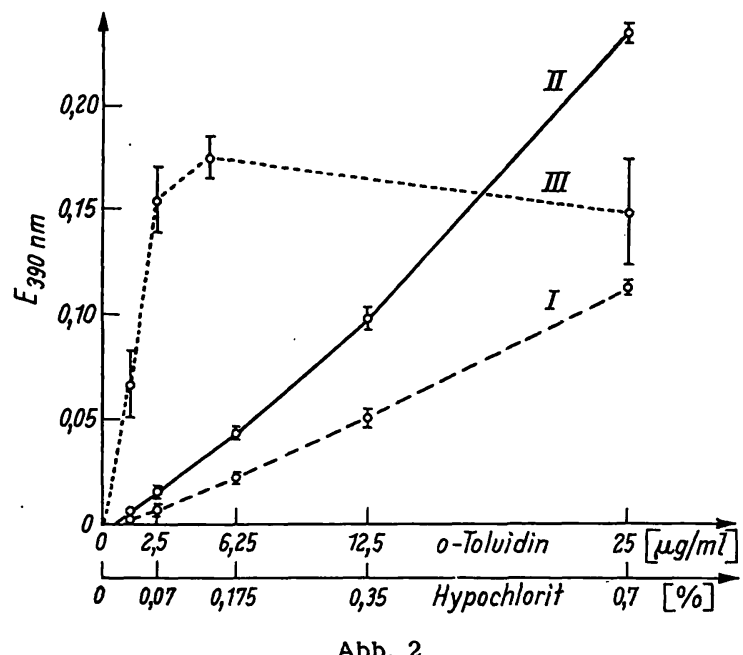

Abb. 2

Eichkurven zur o-Toluidinbestimmung für $1 \mathrm{~cm}$ (Kurve I) und $2 \mathrm{~cm}$ Schichtdicke (Kurve II) bei $390 \mathrm{~nm}$ und Abhängigkeit der Farbbildung von der Konzentration des zugesetzten Hypochlorits (Kurve III)

entwickelnde gelbe Farbe wird nach $30 \mathrm{Min}$. bei $390 \mathrm{~nm}$ photometrisch gemessen. Die Eichkurve ist in dem angegebenen Bereich nahezu linear (Abb. 2). Die Intensität der Farbbildung ist von der Hypochloritkonzentration abhängig (Abb. 2, Kurve III). Daher ist diese bei jeder Serie gleichzuhalten.

\section{Toxizitätsversuche}

Zur Prüfung der Toxizität des 0 -Toluidins verwendeten wir weiße Mäuse (Händlertiere) ron 15-20 g Gewicht beiderlei Geschlechts, die gemischte Stallkost und Wasser ad libitum erhielten.

Wir prüften sowohl die Toxizität bei subkutaner Injektion als auch die Hautresorption. Dazu trugen wir auf eine etwa $1 \mathrm{~cm}^{2}$ große, mit Bariumsulfid enthaarte Hautpartie des Nackens 0 -Toluidin in verschiedenen Lösungsmitteln auf. Als $\mathrm{Maß}$ für die toxische Wirkung bestimmten wir den Met-Hämoglobin-Gehalt. Die Tiere wurden dekapiert und $0,2 \mathrm{ml}$ des aufgefangenen Blutes zur MetHämoglobin-Bestimmung nach der von RICHTERICH (9) angegebenen Vorschrift der Methode von Evelyn und Malloy (10) verwendet.

\section{Lösungen des 0 -Toluidins}

Zur Blutzuckerbestimmung werden 5-10proz. Lösungen von o-Toluidin in Eisessig benutzt. Wir verwenden in unserem Labor das handelsübliche „Dextrogens“ (Feinchemie Sebnitz), das 6\% $o$-Toluidin enthält. Für Vergleichszwecke lösten wir 0 -Toluidin in Glycerin oder iso-Propanol, da die organische Base nur gering wasserlöslich ist. Dagegen sind die Salze des o-Toluidins mit Säuren (z. B. Salzsäure oder Essigsäure) gut wasserlöslich. Für unsere Tropfversuche an der Mäusehaut haben wir neben dem 0 Toluidin-Eisessig-Reagenz wegen der stark ätzenden Wirkung des Eisessigs u. a. eine Gproz. Lösung in 4,5proz. (v/v) Essigsäure, die mit Natronlauge auf pH 3,7 eingestellt worden war, benutzt.

\section{Ergebnisse}

Von Bedeutung für die Praxis ist die Menge von 0 Toluidin, die bei den Bedingungen der Blutzuckerbestimmung ( 8 Min. langes Erhitzen im Wasserbad, Füllung der Reagenzgläser etwa $4 \mathrm{~m} /$ ) in die Luft entweicht. In Tabelle 1 ist diese Menge mit Lösungen von o-Toluidin als reine Base bzw. in verschiedenen Solventien verglichen. Unter den Standardbedingungen lag die 0 -Toluidinabgabe unter der Nachweisgrenze der verwendeten Methode (Serie V). Bei Verdopplung der Erhitzungsdauer (16 Min.) fanden wir jedoch bereits meßbare Mengen (Serie IV). Noch mehr o-Toluidin entweicht, wenn man ein o-Toluidin-Eisessig-Wasser-
Tab. 1

Flüchtigkeit von 0 -Toluidin aus Gemischen mit verschiedenen Lösurigsmitteln beim Erhitzen im siedenden Wasserbad. Versuchsanordnung siehe Abbildung 1, Füllmenge der Reagenzgläser $4 \mathrm{ml}$, Erhitzungsdauer' 8 Min., bei (IV) 16 Min.

\begin{tabular}{llcc}
\hline & Lösung & $\cdots$ & $\begin{array}{c}\text { freigesetztes } \\
\text { o-Toluidin }(\mu \mathrm{g} \pm \mathrm{s})\end{array}$ \\
\hline (I) & o-Toluidin (unverdünnt) & 4 & $791 \pm 246$ \\
(II) & o-Toluidin (5proz. in Glycerin) & 4 & $410 \pm 119$ \\
(III) & 0-Toluidin: Eisessig (1:2) (v/v) & 4 & $87 \pm 43$ \\
(IV) & (6proz. in Eisessig) & 4 & $23 \pm 21$ \\
(V) & (6proz. in Eisessig) & 4 & $2 \pm-$ \\
\hline
\end{tabular}

Gemisch verwendet, das einen Überschuß von $5 \%$ $o$-Toluidin (entsprechend etwa $0,3 \%$ freies Toluidin) enthält (Serie III). Bei 5\% o-Toluidin in Glycerin bzw reinem $o$-Toluidin (Serien II bzw. I) sind es mit über 400 bzw. $800 \mu \mathrm{g}$. schon erhebliche Mengen.

Injiziert man Mäusen $0,01-0,03 \mathrm{~m} l$ reines $o$-Toluidin unter die Rückenhaut, so findet man nach einer halben Stunde die Tiere gelähmt mit zeitweiligen Zuckungen und hechelnder Atmung. Die Dosis $0,01 \mathrm{~m} l$ wird von $100 \%$ der Tiere überlebt, während bei den Dosen 0,02 und $0,03 \mathrm{ml}$ etwa $30 \%$ in diesem akuten Stadium eingehen. Der Rest erholt sich jedoch erstaunlich rasch und nach 1-2 Stunden sind die Tiere wieder mobil und am nächsten Tage erscheinen sie völlig unauffällig. Die Verträglichkeit so großer Dosen (auf $70 \mathrm{~kg}$ umgerechnet entspräche dies $60 \mathrm{ml}$ o-Toluidin s. c.) ließ uns auf Grund der fehlenden praktischen Bedeutung auf die Bestimmung der $\mathrm{LD}_{50}$ acuta verzichten. Statt dessen haben wir bei mehreren Serien die Met-HämoglobinBildung gemessen.

Wie Tabelle 2 zeigt, liegt nach $0,01 \mathrm{~m} l$ Toluidin s. c. der Met-Hämoglobin-Wert in Abhängigkeit von der Zeit

Tab. 2

Met-Hämoglobin-Gehalt im Blut von Mäusen nach subkutaner Injektion von 0 -Toluidin (reine Base)

\begin{tabular}{|c|c|c|c|}
\hline $\begin{array}{l}\text { o-Toluidin } \\
\text { ml injiziert }\end{array}$ & Tötung nach & $\mathbf{n}$ & Met-Hb $(\% \pm \mathrm{s})$ \\
\hline$\dot{-}$ & 一 & 4 & $2,1 \pm 0,9$ \\
\hline 0,01 & $1 \mathrm{Std}$. & 4 & $10,0 \pm 3,1$ \\
\hline 0,01 & 2,5 Stdn. & 4 & $6,9 \pm 1,9$ \\
\hline 0,01 & 19 Stdn. & 9 & $8,6 \pm 2,4$ \\
\hline 一 & - & 4 & $1,2 \pm 0,6$ \\
\hline 0,01 & 3 Tagen & 4 & $6,6 \pm 2,4$ \\
\hline 0,02 & 3 Tagen & 2 & $11,5 \pm-$ \\
\hline 0,03 & 3 Tagen & 3 & $13,2 \pm 5,3$ \\
\hline 一 & 20 Stdn. & 4 & $2,8^{\circ} \pm 0,9$ \\
\hline $\begin{array}{l}0,016 \text { proz. in } \\
\text { Isopropanol }\end{array}$ & 20 Stdn. & 3 & $3,3 \pm 0,7$ \\
\hline
\end{tabular}

zwischen Injektion und Tötung zwischen 10,0\% (1 Stunde später) und 6,6\% (3 Tage später). Bei den höheren Dosen $(0,02$ und $0,03 \mathrm{~m})$ ist die Met-Hämoglobin-Bildung noch größer, erreicht jedoch trotz des vorangegangenen fast letalen Zustandes bei weitem nicht den in der Literatur angegebenen Wert von $60 \%$ (5). Tropft man den Tieren Toluidin auf die Haut, so findet man nach einmaliger Dosis (Versuch I, Tab. 3) von $0,01 \mathrm{ml}$ keine Erhöhung 'des Met-HämoglobinSpiegels über den Normalwert, der bei den Mäusen bis 
3,5\% Met-Hämoglobin gerechnet werden muß. Erhalten die Tiere 6 Tage täglich $0,01 \mathrm{~m} /$ aufgetropft und am Tage vor der Tötung $3 \mathrm{mal} 0,01 \mathrm{~m}$, so beträgt der Met-Hämoglobin-Gehalt des Blutes $16,7 \%$. Bemerkenswert ist, daß die. Kontrolltiere im gleichen Stall einen Met-Hämoglobin-Gehalt von $4,2 \%$ zeigen. Diese geringgradige Erhöhung dürfte entweder auf einer Toluidin-Zufuhr durch Einatmung oder mit größerer Wahrscheinlichkeit auf einer oralen Zufuhr (Lecken) beruhen. Liegt das o-Toluidin als Salz der Essigsäure vor (Überschuß von Essigsäure) (IV), so findet man nach 1 Woche Toluidin-Applikation keine signifikante Erhöhung des Met-Hämoglobin-Gehaltes gegenüber den Kontrollen (V). Ebenso verhält es sich beim Auftropfen der Original-Reagenzlösung „Dextrogens (VI) gegenüber Kontrollen im gleichen Stall (VII). Der Eisessig führte an der zarten Mäusehaut zu sehr starken Nekrosen und Schorfbildung, so daß die Versuche bereits nach dreimaliger Dosis (3 Tagen) beendet werden mußten. Auch wenn die Dextrogens-Lösung nur 5 Minuten einwirken konnte und danach mit Wasser abgespült wurde, traten bereits Nekrosen ein. Eine Erhöhung des Met-Hämoglobin-Gehaltes fand sich jedoch nicht (Gruppe VIII).

Als letzte Serie haben wir das o-Toluidin als 6proz. Lösung in iso-Propanol aufgetragen (Gruppe IX). Eine statistisch signifikante Erhöhung gegenüber der mit iso-Propanol behandelten Kontrollgruppe (X) trat nicht auf.

Der Met-Hämoglobin-Gehalt der Laborantin, die täglich Blutzucker mit der o-Toluidin-Methode bestimmt, lag bei mehrmaligen Kontrollen unter $1 \%$ und damit im Normbereich von $0,2-1 \%$.

\section{Diskussion}

Die mitgeteilten Befunde zeigen in aller Deutlichkeit, $\mathrm{da} ß$ eine Gesundheitsgefährdung des Laborpersonals durch o-Toluidin bei der Blutzuckerbestimmung nicht zu erwarten ist.

Eine Verflüchtigung aus Eisessig ist in nennenswertem Maße nicht festzustellen, wie nach der Erfahrung mit anderen organischen Basen zu erwarten war (11, 12, vgl. auch 13). Selbst von der reinen Base geht pro Ansatz während der 8 minutigen Kochdauer nur etwa $0,5 \mathrm{mg}$ in die Luft über. $\mathrm{Da}$ die maximal zulässige Arbeitsplatzkonzentration (MAK) für 8stündige Beschäftigung $19 \mathrm{mg}$ pro $\mathrm{m}^{3}(=5 \mathrm{ppm})$ beträgt, könnten also pro $\mathrm{m}^{3}$ Laborluft 20 Reagenzgläser mit der reinen Toluidinbase (!) ohne Gefährdung für das Personal gekocht werden. Aus der essigsauren Reagenzlösung wird jedoch so wenig Toluidin flüchtig, daß noch nicht $1 \%$ des MAK-Wertes erreicht wird. Tatsächlich ist die Gefährdung aber noch viel kleiner, da man die Reagenzgläser zur Vermeidung des Rücklaufs von Kondenswasser mit Zellstoff o. ä. bedeckt und damit die Verflüchtigung"weiter erheblich herabsetzt.

Zur Frage der Resorption des o-Toluidins durch die Haut ist zunächst festzustellen, daß schwere Ver- giftungen durch 0 -Toluidin beschrieben sind $(12,14)$. Dabei handelte es sich jedoch durchweg um die freie Base. Es ist bekannt, daß durch die unverletzte Haut nur öllösliche Stoffe aufgenommen werden (siehe z. B. $15,16)$. In saurer Lösung liegt das 0 -Toluidin aber als nicht-öllösliches Salz vor (o-Toluidinacetat dissoziiert in $\mathrm{C}_{6} \mathrm{H}_{4} \mathrm{CH}_{3} \mathrm{NH}_{3}^{+}$und Säureanion). Eine Aufnahme ist deshalb in nennenswertem Umfang nicht möglich, wie unsere Versuche indirekt beweisen. Die gleiche Auffassung wird in der Literatur für das analoge Anilin vertreten (12). Zur Behandlung von Hautverunreinigungen mit Toluidin wird aus diesen Gründen Essigsäure empfohlen (siehe z. B. 17). Selbst durch die mit Schorf bedeckte verletzte Haut scheint 0 -Toluidin in Eisessig nicht erheblich einzudringen (Tab. 3, Versuch

Tab. 3

Met-Hämoglobin-Gehalt im Blut von Mäusen nach täglicher Hauttropfung von o-Toluidin in verschiedenen Lösungsmitteln. Menge
jeweils $0,01 \mathrm{ml}$. Die Kontrollen jeder Serie wurden im gleichen Stall gehalten und nur mit Lösungsmittel getropft

\begin{tabular}{|c|c|c|c|c|}
\hline & Tropfung mit & Tage & $\mathrm{n}$ & $\begin{array}{l}\text { Met-Hb } \\
(\% \pm s)\end{array}$ \\
\hline (I) & 0 -Toluidin & 1 & 10 & $2,2 \pm 1,0$ \\
\hline (III) & $\begin{array}{l}\text { o-Toluidin } \\
\text { Kontrolle }\end{array}$ & $7 *$ & $\begin{array}{l}7 \\
4\end{array}$ & $\begin{array}{r}16,7 \pm 1,8 \\
4.2 \pm 0,3\end{array}$ \\
\hline \multirow{2}{*}{$\begin{array}{l}\text { (IV) } \\
\text { (V) }\end{array}$} & \multirow{2}{*}{$\begin{array}{l}\text { o-Toluidin (6proz. in Essigsäure } \\
\text { pH } 3,7 \text { ) } \\
\text { Kontrolle (Essigsäure-Acetat } \\
\text { pH } 3,7 \text { ) }\end{array}$} & $7 *$ & 8 & $3,5 \pm 0,5$ \\
\hline & & $7 *$ & 8 & $3,1 \pm 0,7$ \\
\hline $\begin{array}{l}\text { (VI) } \\
(\mathrm{VII})\end{array}$ & $\begin{array}{l}\text { "Dextrogens" } \\
\text { Kontrolle (Eisessig) }\end{array}$ & $\begin{array}{l}3 \\
3\end{array}$ & $\begin{array}{l}6 \\
4 \\
\end{array}$ & $\begin{array}{l}5,0 \pm 1,8 \\
4,5 \pm 1,4\end{array}$ \\
\hline (VIII) & Dextrogens (abgespült) & 2 & 7 & $2,7 \pm 0.8$ \\
\hline $\begin{array}{l}(\mathrm{IX}) \\
(\mathrm{X})\end{array}$ & $\begin{array}{l}\text { c-Toluidin (6proz. in iso- } \\
\text { Propanol) } \\
\text { Kontrolle (iso-Propanol) }\end{array}$ & $\begin{array}{l}7 * \\
7 *\end{array}$ & $\begin{array}{l}8 \\
8\end{array}$ & $\begin{array}{l}3,1 \pm 1,0 \\
3,6 \pm 0,5\end{array}$ \\
\hline$(\mathrm{XI})$ & Kontrolle (unbehandelt) & 一 & 11 & $3,3 \neq 0,8$ \\
\hline
\end{tabular}

* Am Tage vor der Tötung $3 \mathrm{mal}$ täglich $0,01 \mathrm{ml}$.

VI). Bei der Laborarbeit wird im allgemeinen durch das Brennen des Eisessigs ein längerer Hautkontakt mit dem Reagenz zur Blutzuckerbestimmung vermieden werden. Zusammenfassend können wir daher die von BürGI und MrtTELHolzer (5) postulierte Gesundheitsgefährdung durch das bei der Blutzuckerbestimmung benutzte o-Toluidin-Eisessigreagenz ablehnen. Selbstverständlich ist zu beachten, daß bei der kaum rentablen Selbstzubereitung der Reagenzlösung aus der freien Base Vorsicht zu walten hat. Insbesondere muß eine meist notwendige Destillation der o-Toluidin-Base in jedem Falle unter dem Abzug erfolgen. Auch soll in diesem Zusammenhang nicht unerwähnt bleiben, $\mathrm{da} B \operatorname{der}$ MAK-Wert für Eisessig nur das 4-5fache des oToluidins beträgt $\left(25 \mathrm{ppm}=65 \mathrm{mg}\right.$ pro $\left.\mathrm{m}^{3}\right)(7)$. Bei -der reichlichen Verwendung von Eisessig im klinischen Labor erscheint ein Hinweis auf die nötige Vorsicht auch mit diesem Reagenz angebracht.

Anmerkung bei der Korrektur:

Während der Drucklegung erschien eine Arbeit ron A. HärTeL und Mitarbeiter (diese Z. 7, 183 (1969)), in der mit anderen Methoden ebenfalls die praktische Ungefährlichkeit der o-ToluidinEisessig-Methode zur Blutzuckerbestimnung nachgewiesen wurde. 


\section{Literatur}

1. Hultman, E., Nature (London) 183, 108 (1959). - 2. Duвowskr, K. M., Clin. Chem., New York 8, 215 (1962). - 3. HYvärINEN, A. und E. A. NIKkILÄ, Clin. chim. Acta, Amsterdam 7, 140 (1962). - 4. AhLert, G., E. Hofer, W. HoffmanN und G. Bestvater, Dtsch. Ges.wesen 19, 2256 (1964). - 5. Bürgr, W. und M. L. Mrttelholzer, Praxis 57, 3 (1968). - 6. Houben, J., Fortschritte der Heilstoffchemie. 2. Abt., II. Bd., S. 829, Walter de Gruyter, Berlin (1932). - 7. WITRH, W., G. HECHT und $\mathrm{C}_{\mathrm{H}}$. Gloxhuber, Toxikologie-Fibel, G. Thieme, Stuttgart (1967). 8. Bauer, $H$. und H. Molr, Die organische Analyse. 4. Auflage, Akadem. Verlagsgesellschaft Geest \& Portig, Leipzig (1960). 9. Richterich, R., Klinische Chemie. S. Karger-Verlag, BaselNew York (1965). - 10. EvelyN, K. A. und H. T. MaLioy, J. biol. Chemistry 126, 655 (1938), zit. nach (9). - 11. Gemeinhard,
K., Nachweis wichtiger Arzneimittel und Gifte. In: Hoppe-SeylerThierfelder: Handbuch der physiologisch- und pathologischchemischen Analyse. 10. Auflage, Bd. 5, S. 760, Springet-Verlag, Berlin-Göttingen-Heidelberg (1953). $\because$ 12. LEHMANN, K. B., Kurzes Lehrbuch der Arbeits- und Gewerbehygiene. HirzelVerlag, Leipzig (1919). - 13. GADAMER, J., Lehrbuch der chemischen Toxikologie und Anleițung zur Ausmittlung der Gifte. Vandenhoeck \& Ruprecht, Göttingen (1924). - 14. LEwrN, L., Gifte und Vergiftungen. G. Stilke-Verlag, Berlin (1929). - 15. HausCHILD, F., Pharmakologie und Grundlagen der Toxikologie, 2. Aufl., G. Thieme, Leipzig (1960). - 16. Bürgr, E., Die Durchlässigkeit der Haut für Arzneien und Gifte, Springer-Verlag, Berlin (1942). - 17. Ludewrg, R. und K. Lors, Akute Vergiftungen. G. Fischer-Verlag, Jena (1966).

ao. Doz. Dr. W.-D. Thomitzek und Dr. H. Bemm, Stadtkrankenhaus Friesenstraße 7033 Leipzig, Friesenstr. 8 